\title{
1 An improved X-ray diffraction method for cellulose crystallinity measurement
}

3 Xiaohui Ju', Mark Bowden ${ }^{\dagger}$, Elvie E Brown ${ }^{\dagger}$, Xiao Zhang ${ }^{\dagger *}$

4

$5{ }^{\dagger}$ School of Chemical Engineering and Bioengineering, Washington State University, Richland,

6 Washington, 99354, United States

7 Environmental Molecular Science Laboratory, Pacific Northwest National Laboratory, Richland,

8 Washington, 99352, United States

9

10

11

12

13

14

$15 *$ Corresponding authors:

16 E-mail: x.zhang@wsu.edu; Telephone: 1-509-372-7647; Address: Bioproducts, Sciences \&

17 Engineering Laboratory, 2710 Crimson Way, Richland, WA, 99354.

18 E-mail: Mark.Bowden@ @nnl.gov; Telephone: (509) 371-7816

19

20

21

22

23 


\section{Abstract}

25 We show in this work a modified X-ray diffraction method to determine cellulose crystallinity

26 index (CrI). Nanocrystalline cellulose (NCC) dervided from bleached wood pulp was used as a

27 model substrate. Rietveld refinement was applied with consideration of March-Dollase preferred

28 orientation at the (001) plane. In contrast to most previous methods, three distinct amorphous

29 peaks identified from new model samples which are used to calculate CrI. A 2 theta range from

$3010^{\circ}$ to $75^{\circ}$ was found to be more suitable to determine $\mathrm{CrI}$ and crystallite structural parameters

31 such as d-spacing and crystallite size. This method enables a more reliable measurement of $\mathrm{CrI}$

32 of cellulose and may be applicable to other types of cellulose polymorphs.

\section{$36 \quad$ Keywords}

37 Crystallinity index, nanocrystalline cellulose, X-ray diffraction, cellulose crystallite, cellulose 38 polymorphs 


\section{Introduction}

The presence of crystallinity in cellulose is one of the most important characteristics contributing to its physical, chemical and mechanical properties (Andersson, Serimaa, Paakkari,

50 Saranpaa \& Pesonen, 2003; Ryu, Lee \& Tassinari, 1981; Soltes, 1983; Tanahashi, Goto, Horii,

51 Hirai \& Higuchi, 1989; Weimer, Hackney \& French, 1995). Crystallinity index (CrI) is a

52 parameter commonly used to quantify the amount of crystalline cellulose present in cellulosic

53 materials and has also been applied to interpret changes in cellulose structures after

54 physicochemical and biological treatments (Ai-Zuhair, 2008; Andersson et al., 2003; Cao \& Tan,

55 2005; Chanzy, Marechal \& Nishiyama, 1999; Hall, Bansal, Lee, Realff \& Bommarius, 2010).

56 Analytical methods to determine $\mathrm{CrI}$ include ${ }^{13} \mathrm{C}$ nuclear magnetic resonance (NMR)

57 spectroscopy, Fourier transform (FT)-IR spectroscopy, and X-ray diffraction (XRD). Among

58 these, XRD is the most prevailing method currently employed (Agarwal, Reiner \& Ralph, 2010;

59 Bansal, Hall, Realff, Lee \& Bommarius, 2010; Deraman, Zakaria \& Murshidi, 2001; Evans,

60 Newman, Roick, Suckling \& Wallis, 1995; Kljun, Benians, Goubet, Meulewaeter, Knox et al. ,

61 2011; Liitia, Maunu \& Hortling, 2000; Liitia, Maunu, Hortling, Tamminen, Pekkala et al. , 2003;

62 Park, Baker, Himmel, Parilla \& Johnson, 2010; Teeaar, Serimaa \& Paakkari, 1987).

There are three methods commonly applied to calculate the CrI of cellulose based on the

64 XRD data (Bansal et al., 2010; Cao \& Tan, 2005; Driemeier \& Calligaris, 2011; French \&

65 Santiago Cintron, 2013; Park et al., 2010; Rietveld, 1969; Rowe, Mckillop \& Bray, 1994;

66 Ruland, 1961; Segal, Creely, Jr \& Conrad, 1962; Tanahashi et al., 1989; Thygesen, Oddershede,

67 Lilholt, Thomsen \& Stahl, 2005). The first was developed by (Segal et al., 1959), based on the

68 ratio of the height of the (200) peak $\left(\mathrm{I}_{200}\right)$ and the height of the minimum $\left(\mathrm{I}_{\mathrm{AM}}\right)$ between the (200)

69 and (110) peaks. This is the simplest and most frequently used technique. However, as the exact 
70 amount of crystalline fraction is proportional to the peak area rather than its height, the peak

71 height calculation presents an obvious flaw to accurate measurement of CrI. In addition, a recent

72 study (French \& Santiago Cintron, 2013) has shown that the CrI obtained using this method is

73 dependent on crystallite size and cellulose polymorph, for materials with the same fraction of

74 crystalline cellulose. The second method is based on peak deconvolution of crystalline and

75 amorphous peaks. The crystalline cellulose is represented by several intense peaks at (1ī0), (110),

76 (102), (200), and (004) for cellulose $I_{\beta}$. The positions and areas of these peaks may be arbitrarily

77 chosen (Garvey, Parker \& Simon, 2005) or calculated from a known crystal structure. The

78 amorphous fraction is typically modeled by a single broad peak and $\mathrm{CrI}$ is calculated from the

79 ratio of the area of the crystalline peaks to the total area. The accuracy of this method is

80 challenged by the difficulty in selecting peaks that correctly correspond to the actual diffraction

81 contributed by each fraction. The third common technique is the amorphous subtraction method

82 first described by Ruland (Ruland, 1961) and later modified by Vonk (Vonk, 1973). The method

83 fits an intensity profile of an amorphous component which is scaled so that it remains just below

84 all the observed intensity from the experimental sample pattern. The $\mathrm{CrI}$ is determined from the

85 ratio of the area above the amorphous profile to the total area. The amorphous profile is obtained

86 either from a polynomial function or a pattern measured from experimentally prepared material

87 believed to be entirely amorphous (e.g., ball-milled cellulose, regenerated cellulose, xylan or

88 lignin powder) (Bansal et al., 2010; Thygesen et al., 2005).

89 Detailed review of these methods is given by (Park et al., 2010). These wide angle X-ray

90 scattering (WAXS) based methods are reliable and commonly used for comparative

91 determination of $\mathrm{CrI}$ between the cellulosic samples from same sources. Yet there is still a

92 challenge in quantification and comparative analysis of CrI among cellulose samples obtained 
93 from different origins and process methods. The crystallites of natural celluloses are small in size

$94(<10 \mathrm{~nm})$ compared to typical crystalline solids like metals or ceramics. Because of the small

95 crystallites and the variations in crystalline structures within cellulose chains, XRD analyses of

96 cellulose always result in broad diffraction peaks which are difficult to distinguish from the

97 diffractions of amorphous cellulose and background scattering. This is the main reason behind

98 the long-term-challenge to apply XRD to obtain an exact quantification of cellulose CrI,

99 especially when comparing samples from different origins or sources.

100 Recognizing the lack of accurate crystallinity measurement method, Driemeier and

101 Calligaris (Driemeier \& Calligaris, 2011) have recently developed new protocol to improve CrI

102 measurement by systematically considering preferred fiber orientation, incoherent scattering,

103 moisture, and other compositional deviations. However, this method is based on transmission

104 data from capillary samples which is a relatively uncommon instrument set up. It requires a

105 separate measurement of an empty capillary and uses a complex background which could

106 substitute for amorphous cellulose. The objective of this study is to modify existing XRD

107 procedures to improve the reliability of WAXS for CrI determination using general XRD

108 instrumentation. The Rietveld method was chosen due to its broad applicability to all cellulose

109 polymorphs with an available crystal structure and its consistency in treating all the crystalline

110 peaks. Although we used commercial Rietveld software in this study, the same functionality is

111 widely available in a range of other programs, some of which are available free of charge. The

112 following approaches were taken in this study: 1) select the model substrates which closely

113 represent the nanolevel size cellulose crystallites and amorphous cellulose; 2) determine the

114 diffraction background in the absence of amorphous material; 3) determine a suitable $2 \theta$ range

115 for better coverage of amorphous and crystalline diffraction. 


\section{2. Material and methods}

\section{$117 \quad 2.1$ Substrate preparation}

118 The brown stock kraft pulp was first prepared from poplar wood chips by cooking with a 119 mixture of $\mathrm{NaOH}$ and $\mathrm{Na}_{2} \mathrm{~S}$ solution at $170^{\circ} \mathrm{C}$ for 160 minutes. Bleached kraft pulp (BKP) was 120 prepared by subsequent treatment of brown stock kraft pulp using acid sodium chlorite solution 121 at room temperature for $24 \mathrm{~h}$ following a procedure previously described (Ju, Engelhard \& 122 Zhang, 2013). Nanocrystalline cellulose was prepared by acid extraction of BKP (Hamad \& Hu, 123 2010). In brief, $64.5 \%$ sulfuric acid was used to extract nanocrystalline cellulose from BKP, 124 followed by dilution to stop reaction. The mixture was filtered through $3 \mathrm{~K}$ Dalton RC membrane 125 using AMICON ultrafitration 8400 system. Nanocrystalline cellulose collected above the 126 membrane after repeated washing and filtering was freeze-dried for further analysis. 127 Microcrystalline cellulose (Avicel PH101) was purchased from Sigma-Aldrich (St Louis, USA). 128 Phosphoric acid swollen amorphous cellulose was prepared using phosphoric acid treating NCC 129 as well as Avicel following procedure as previously described (Zhang, Cui, Lynd \& Kuang, 130 2006).

\section{$131 \quad 2.2 X R D$}

132 XRD samples were front-loaded into shallow (ca. $0.5 \mathrm{~mm}$ ) wells machined into zero 133 background holders manufactured from off-axis single crystal quartz. XRD patterns were 134 collected using a Panalytical Bragg-Brentano goniometer with $\mathrm{Cu} \mathrm{K} \alpha$ radiation, variable 135 divergence and anti-scatter slits (illuminated length $=10 \mathrm{~mm}$ ), and a post-diffraction 136 monochromator. Data were collected from 10 to either 75 or $140^{\circ} 2 \theta$ (see text), counting for $3 \mathrm{~s}$ 137 at $0.05^{\circ}$ intervals. 
139 ratio between the intensity of the crystalline peak $\left(\mathrm{I}_{200}-\mathrm{I}_{\mathrm{AM}}\right)$ and total intensity $\left(\mathrm{I}_{200}\right)$ after

140 subtraction of the background signal. Nonlinear least squares fitting of the data was carried out

141 using TOPAS (v4.2, Bruker AXS) with the crystalline component calculated from the cellulose

142 I $\beta$ structure published by Nishiyama et al. (2002). Peudo-Voigt line shapes were used for the

143 peaks of both crystalline and amorphous (see later) cellulose. A three-parameter $2^{\text {nd }}$ order 144 polynomial function was used for the background and the degree of crystallinity calculated from

145 the area of crystalline cellulose divided by the total area of crystalline + amorphous cellulose.

146 The measured patterns were used without any corrections or other processing; Lorentz-

147 polarization, absorption, and sample displacement corrections were applied to the calculated

148 patterns. The $d$-spacing were calculated using the Bragg's equation (1) and the crystallite sizes

149 were calculated from the widths of diffraction peaks using the Scherrer equation (2):

$150 \quad n \lambda=2 d \sin \theta$

$151 L=0.9 \lambda /(H \cos \theta)$

152 where $\mathrm{n}$ is an integer; $\lambda$ is the wavelength of incident wave length; $d$ is the spacing between the

153 planes in the atomic lattice, $\theta$ is the angle between the incident ray and the scattering planes; $\mathrm{L}$ is

154 the crystallite size perpendicular to the plane and $\mathrm{H}$ is the full width at half-maximum (FWHM)

155 in radians.

\section{3. Results and discussion}

157 To attain suitable representative peaks for the crystalline component of cellulose, 158 nanocrystalline cellulose (NCC) prepared from bleached wood pulp (Dong, Revol \& Gray, 1998)

159 was used as a model compound in this study. In nature, cellulose does not occur as an individual 160 molecule, it is composed of crystalline and amorphous components. During NCC preparation, 
161 the amorphous regions of cellulose are removed inducing a transverse cleavage of cellulose

162 fibers into rodlike nanoparticles (Habibi, 2014). This transformation consists of disruption of the

163 amorphous regions surrounding the cellulose microfibrils, as well as those embedded between

164 them, while leaving the crystalline segments intact. The NCC fibers are 5-20 nm in width, with

165 length up to $200 \mathrm{~nm}$ based on the measurement by both dynamic light scattering particle size

166 analyzer and atomic force microscope (Ju et al., 2014). This dimension closely represents the

167 unit size of 1-4 cellulose crystallites in a cross section (Ju et al., 2013). These NCC particles

168 closely represent highly pure individual cellulose crystallite.

169 A lack of an appropriate "amorphous" calibration standard is another reason hindering

170 precise measurement of CrI (Fernandes et al., 2011) To generate an amorphous substrate that

171 directly correlates to crystallite counterpart found in a microfibril, phosphoric acid treatment of

172 NCC was applied to produce regenerated amorphous cellulose (RAC). Concentrated phosphoric

173 acid was used in the treatment to dissolve and swell NCC.

174 Clearly distinguishing between the intensity from the sample and background scattering

175 is critical to obtain an accurate measurement of CrI. Typical X-ray background intensity patterns

176 arise from a variety of coherent and incoherent scattering, including Compton scattering, thermal

177 vibrations of atoms in the sample, and interaction of the X-ray beam with air. Inelastic scattering

178 was not significant in this study, since a monochromator was used to filter out radiation scattered

179 at energies different from the incident radiation.

180 Figure 1 illustrates the need to separate the background scattering from other 181 contributions to the XRD data of NCC. In this refinement we followed typical procedures 182 reported in the literature (Thygesen et al., 2005) and used a 10-parameter Chebychev polynomial 183 background. This background has a complex shape which could be attributed to amorphous 
184 cellulose, for example the relatively rapid rise near $30-40^{\circ} 2 \theta$. To see if this was a realistic 185 background, the diffraction pattern of glucose monohydrate was measured under identical 186 conditions (Figure 2). This material has a similar chemical composition to cellulose but is 187 entirely crystalline and thus should reveal the background without any contribution from 188 amorphous material. The result was a smooth background rising from ca. 10 counts/s at $10^{\circ} 2 \theta$ to 189150 counts/s at $100^{\circ} 2 \theta$, which could be modelled satisfactorily with a 2-parameter Chebychev 190 polynomial.

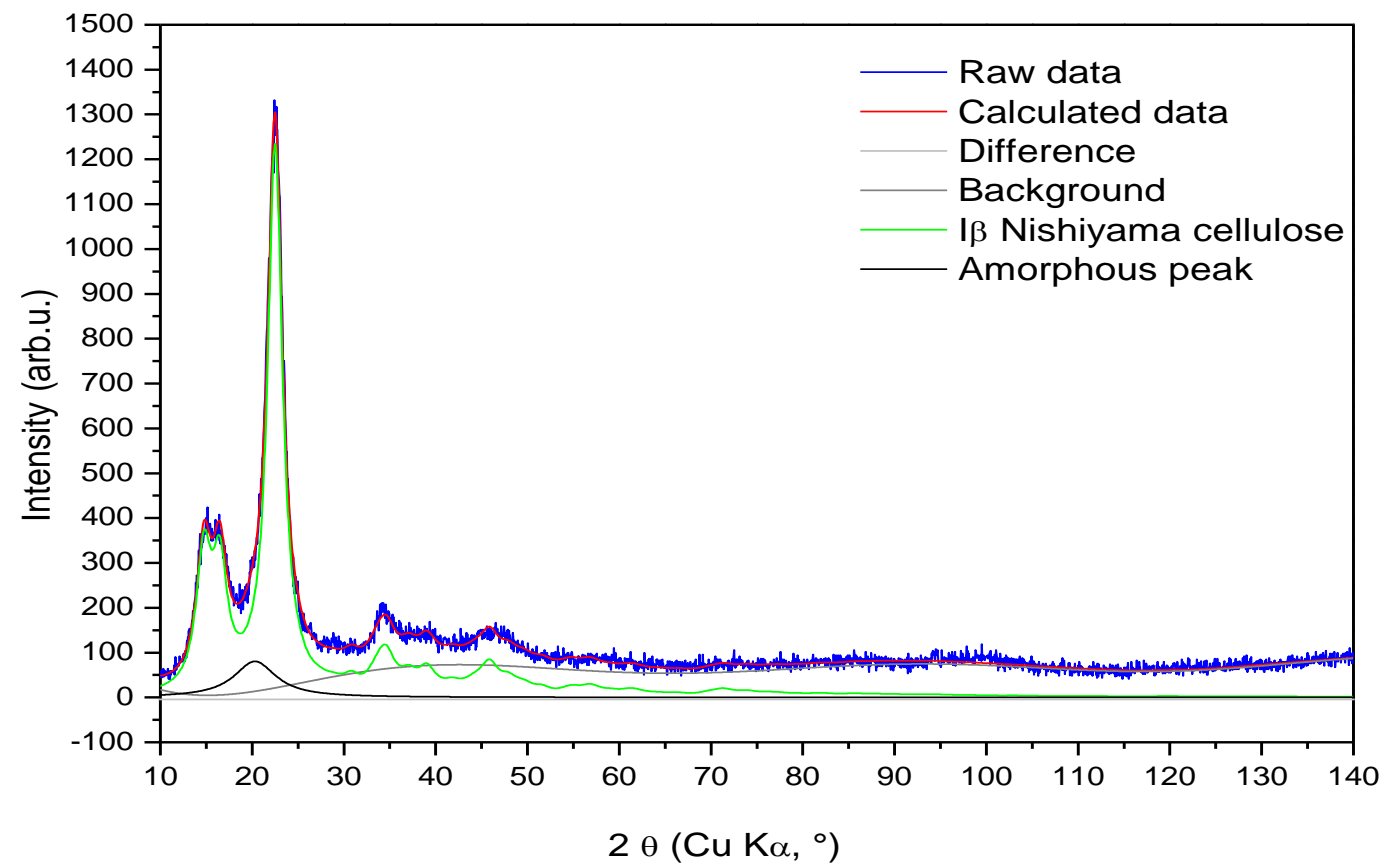

192 Figure 1. Calculated XRD pattern compared to raw data. Rietveld fit of data collected from NCC 193 cellulose. Contributions from crystalline (green) and amorphous (black) cellulose are added to 194 the background (grey) to give the total calculated pattern (red). Nishiyama cellulose is shown to 195 confirm the cellulose peaks. The obtained background was then applied to the pattern measured from RAC, with good 198 results (Figure 3). In this figure, it is clear that amorphous cellulose contributes three broad 199 signals to the diffraction pattern, at approximately $20.5,38.9$, and $80.9^{\circ} 2 \theta$. This is in contrast to 200 previous research which has predominantly used a single amorphous peak centered at $20^{\circ}$ 
201 (Bansal et al., 2010; Driemeier \& Calligaris, 2011; Park et al., 2010). The other two peaks were

202 not considered in the majority of published studies, presumably because the peaks at higher

203 diffraction angle are broader and have lower maxima than the peak near $20^{\circ} 2 \theta$. However since

204 the CrI calculation for peak deconvolution methods relies on areas, ignoring these additional

205 peaks from amorphous cellulose would introduce substantial errors. In our work we used all

206 three peaks to model the amorphous fraction, fixing their positions and widths and constraining

207 their intensities to the same ratio as found in the RAC sample.

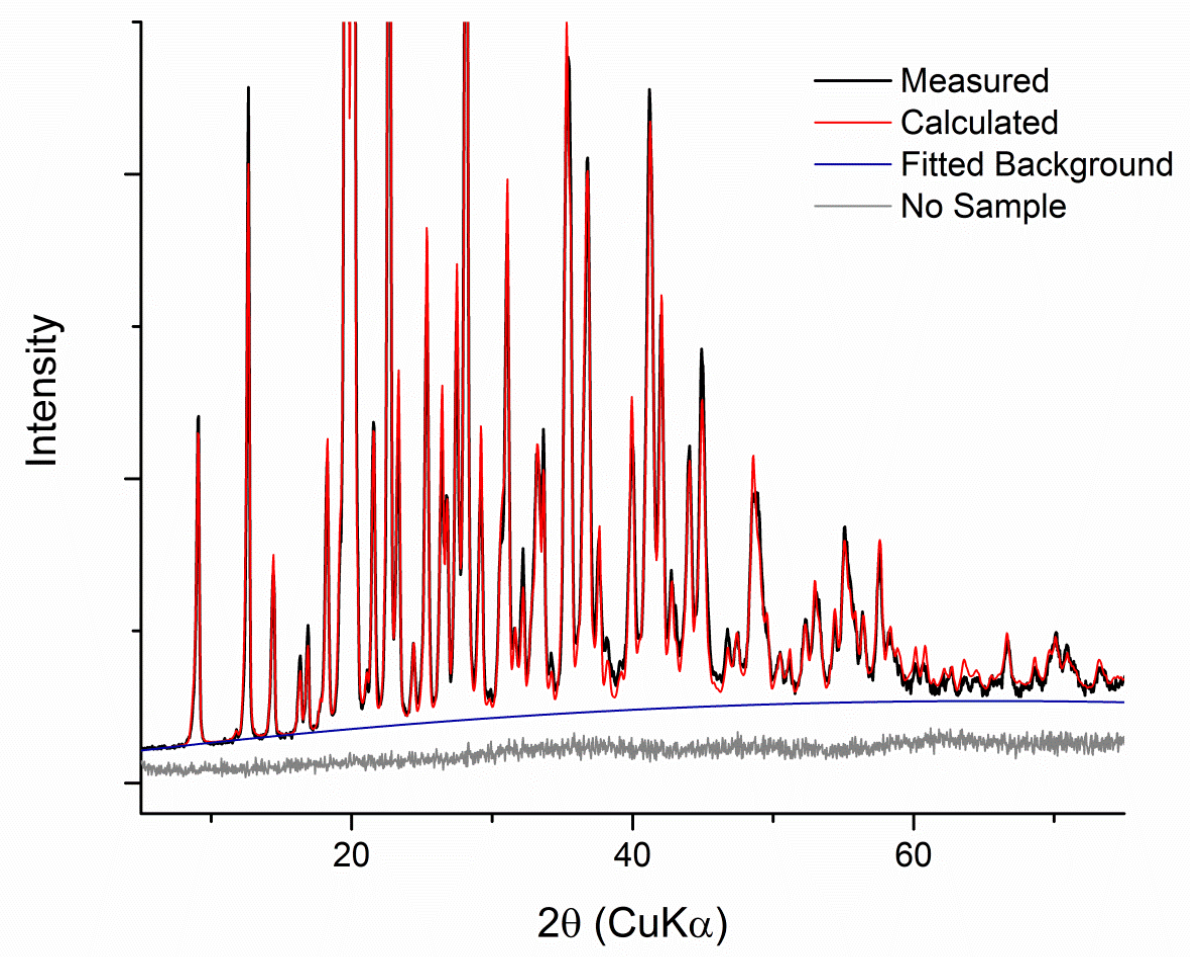

209 Figure 2. Diffraction pattern of crystalline glucose with expanded vertical scale to show the 210 shape of the background. The fitted background is shown in blue and matches the shape of the 211 pattern recorded from an empty sample holder (gray, offset for clarity). These patterns are 212 recorded with a variable divergence slit which increases the $x$-ray flux with in increasing 213 diffraction angle, which will contribute to the background shape. 


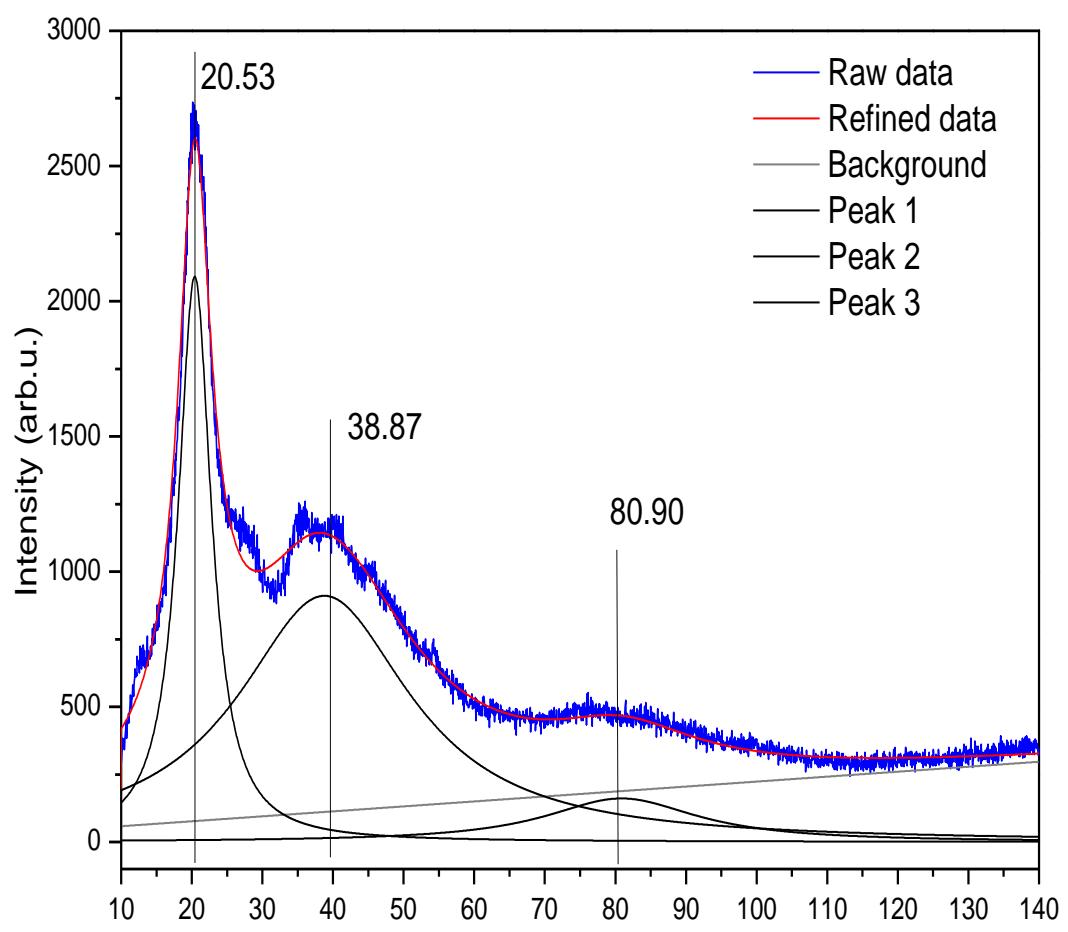

217 Figure 3. Experimentally generated amorphous cellulose material shows broad peaks centered at $21820.53^{\circ}, 38.87^{\circ}$ and $80.90^{\circ}$.

221 crystal structures published by Nishiyama et al, 2002 (Nishiyama, Langan \& Chanzy, 2002).

222 Since cellulose frequently forms in long fibrils, it is important to account for the preferred

223 orientation that is almost invariably present in diffraction samples. The Rietveld program 224 employed contains two computational options which are also widely available in other Rietveld

225 software: 1) a spherical harmonics approach and 2) the March-Dollase approach (Dollase, 1986).

226 After numerous experimentation, it was found that more reliable results were obtained using a

227 single (001) March-Dollase correction. This approach had the advantage of a single refinable 228 parameter and avoided the negative peak intensities which were occasionally observed from 
applying spherical harmonic corrections. A pseudo-Voigt line shape was used to calculate the 230 diffraction peaks.

231 Having established suitable methods for calculating the background intensity and the 232 crystalline and amorphous peaks, we sought a suitable diffraction range to calculate cellulose 233 CrI. This range should cover the significant areas where amorphous and crystalline components 234 diffract, but be sufficiently small to allow reasonable data collection times. Figure 4 shows the $235 \mathrm{XRD}$ data from the crystalline model substrate, NCC, over the range of $10^{\circ}$ to $140^{\circ} 2 \theta$. This 236 wide range should capture all of the intensity from both the amorphous and crystalline fractions.

237 The CrI was determined from the area of the crystalline portion divided by the total of crystalline 238 and amorphous peak areas.

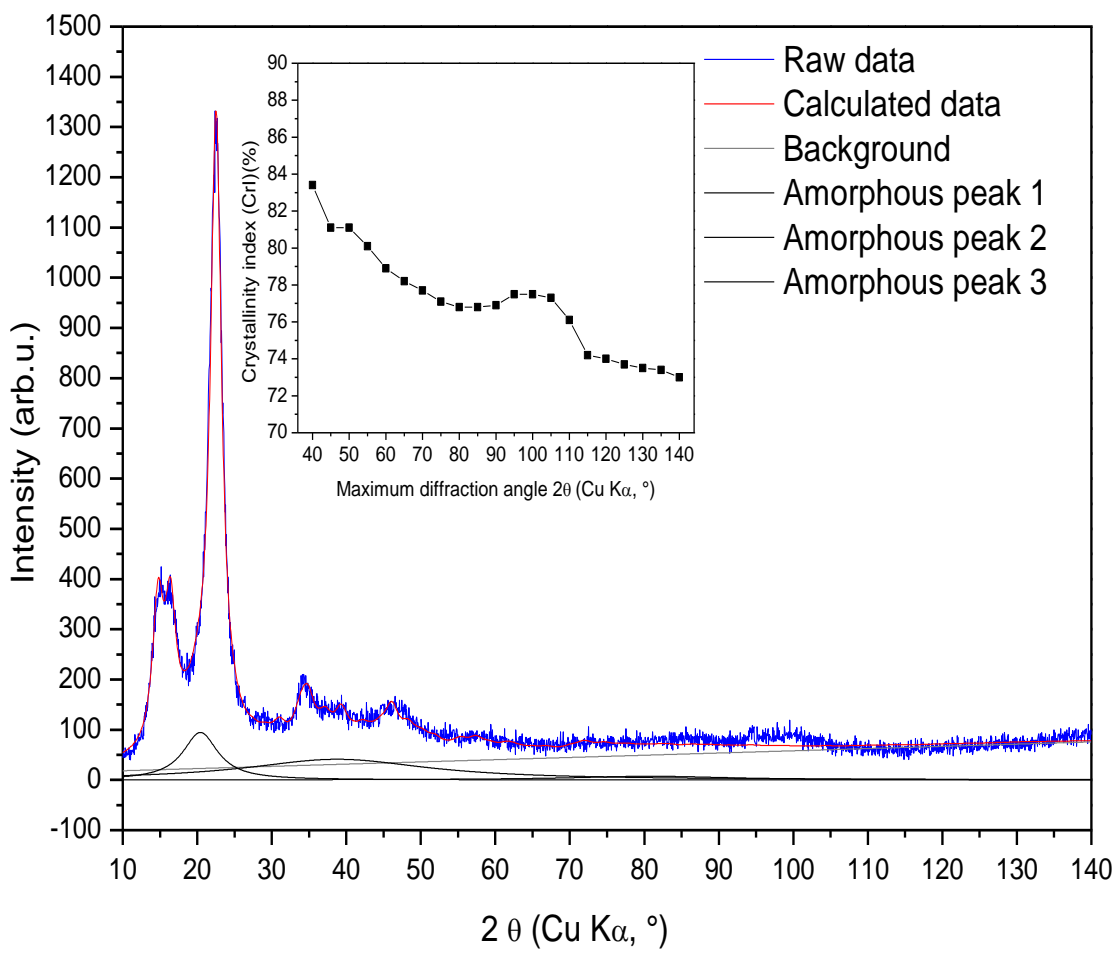

240 Figure 4. Rietveld fit of NCC up to $140^{\circ}$ with three experimentally defined amorphous peaks 241 (Inset: variation of determined CrI vs. the maximum $2 \theta$ angle used for calculation). 
In order to see whether a smaller $2 \theta$ range gives comparable results, the diffraction

244 profile fit and $\mathrm{CrI}$ calculation were repeated as the data were truncated at various diffraction

245 angles. The resulting CrI values are plotted in Figure 4 inset. The estimated fraction of crystalline

246 cellulose remained consistent at approximately $77 \%$ when the diffraction range was from 10 to

$247 \quad 70-90^{\circ} 2 \theta$. When the diffraction range was restricted at less than $70^{\circ} 2 \theta$, the CrI rose, reaching

$24884 \%$ when the range ended at $40^{\circ} 2 \theta$. This is because crystalline cellulose has its most intense

249 peaks below $30^{\circ} 2 \theta$, whereas amorphous cellulose shows broad features with substantial

250 intensity extending beyond $70^{\circ} 2 \theta$ (see Figures 1 and 2). Unexpectedly, CrI dropped when the

251 diffraction range extended past $100^{\circ} 2 \theta$. There was a broad region of fairly weak intensity near

$252100{ }^{\circ} 2 \theta$ in the NCC sample which was not observed in the amorphous sample and did not appear

253 in the pattern calculated from the crystal structure (Figure 3). This unknown intensity affected

254 the Rietveld fit for the larger diffraction ranges, and produced unreliable results.

256 Most of the studies of cellulose CrI examined XRD pattern only to a maximum $2 \theta$ of $50^{\circ}$,

257 partially because the last major crystalline peaks are centered at $35^{\circ}$ (Bansal et al., 2010;

258 Driemeier \& Calligaris, 2011; French \& Cintron, 2013; Hall et al., 2010). However, our findings

259 illustrated that considerable intensity from amorphous cellulose can extend beyond even $70^{\circ}$,

260 making a significant contribution when calculating CrI. Therefore we recommend using a range

261 of $2 \theta$ from $10^{\circ}$ to $75^{\circ}$. This is a practical range that likely covers significant intensities that are 
representative of both crystalline and amorphous components of cellulose.

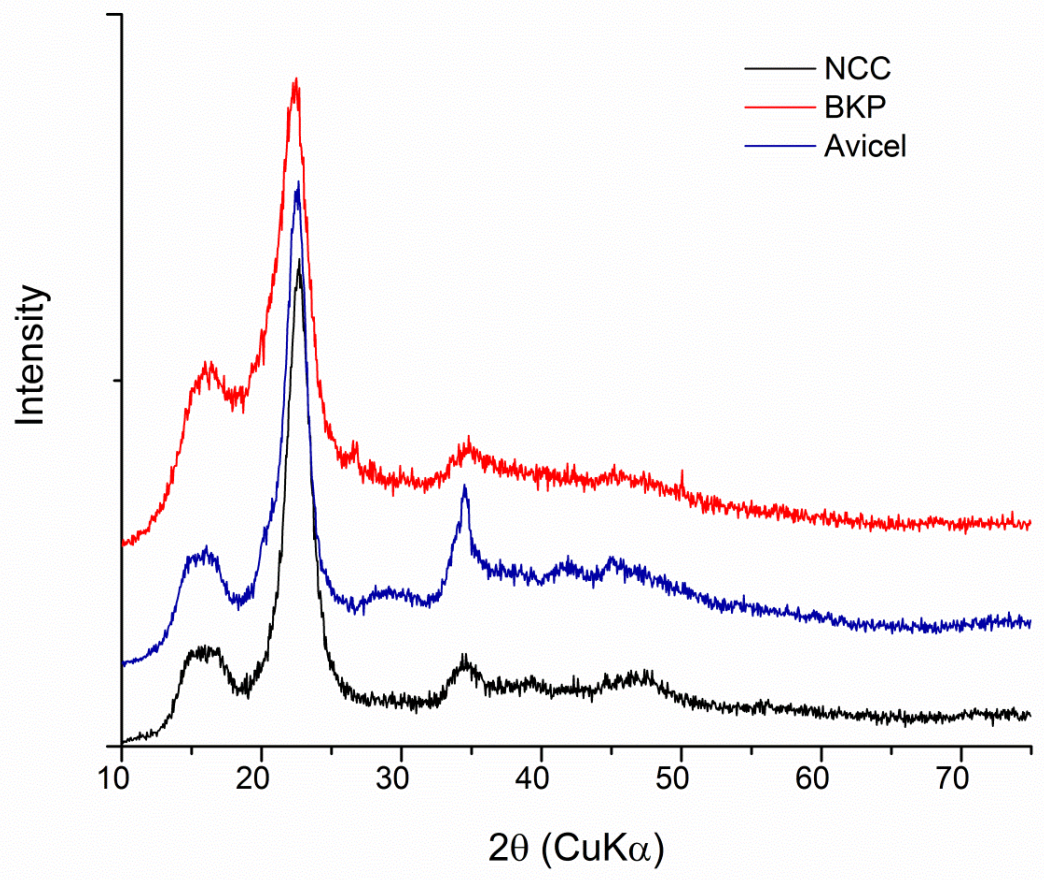

264 Figure 5. Measured XRD patterns of the cellulose samples, scaled to the same maximum 265 intensity and offset for clarity.

To examine the applicability of this method on different cellulose samples, three cellulose

268 model compounds, bleached kraft pulp (BKP) (Ju et al., 2013) prepared from poplar,

269 microcrystalline cellulose Avicel and nanocrystalline cellulose were analyzed. Figure 5 shows

270 the measured XRD patterns of the model compounds, using the improved refinements. Table 1

271 summarizes the CrI values for these samples obtained from this method and common Segal

272 peak-height method. It is well recognized that peak height based CrI overestimates the true

273 crystallinity of cellulose I $\beta$ (Park et al., 2010). As shown in Table 1, lower CrI values were

274 observed for all three tested materials calculated by our peak-fitting method compared to peak

275 height method. . It is noteworthy that the Segal CrI difference between NCC and Avicel is very 
small (90\% vs $88 \%$ ), while our method also showed a significant difference in CrI between NCC

277 and Avicel, by 17\%. This difference is partly due to the sharp (200) peak of NCC which resulted

278 from the highly ordered crystallite structure of NCC. In Avicel, the peak width was decreased

279 without significantly increasing the $\mathrm{I}_{200}$ intensity. The higher peak intensity near $35^{\circ}$ in Avicel

280 also contributed to the lower CrI compared to NCC.

\begin{tabular}{|c|c|c|c|}
\hline Crystallinity index (CrI, \%) & NCC & Avicel & BKP \\
\hline Segal method & $90 \pm 1$ & $88 \pm 1$ & $71 \pm 2$ \\
\hline This method & $77 \pm 3$ & $60 \pm 4$ & $50 \pm 3$ \\
\hline
\end{tabular}

Table 1. Crystallinity index (CrI) calculated for model compounds NCC, Avicel and BKP.

\begin{tabular}{|c|c|c|c|}
\hline & Planes & NCC & Avicel \\
\hline \multirow{3}{*}{$d$-spacing } & $(\overline{1} 10)$ & 0.593 & 0.607 \\
\cline { 2 - 4 } & $(110)$ & 0.531 & 0.544 \\
\cline { 2 - 4 } & $(200)$ & 0.389 & 0.399 \\
\hline \multicolumn{2}{|c|}{ Average crystallite size (nm) } & $4.5 \pm 0.1$ & $5.6 \pm 0.1$ \\
\hline
\end{tabular}

291

Table 2. Crystallite structures of model cellulose compounds calculated from our proposed improved method.

Besides CrI, several key parameters associated with crystallite structure such as 296 crystallite size and d-spacing between glucan chains in the cellulose crystallite were also 297 determined from this method (Table 2). Avicel has been previously used as a model substrate to 298 estimate cellulose crystallite structural parameters. There is little experimental data on crystallite 299 structure of nanocrystallite cellulose. It is apparent that the d-spacings along $\bar{I} 10,110,200$ planes 
300 are all smaller in NCC than Avicel (Table 2). The average particle size of Avicel is in

301 micrometer scale. It is conceivable that cellulose crystallite cores are entwined with amorphous

302 and paracrystalline cellulose layers in Avicel. $d$-spacing value from XRD is the average

303 measurement of entire crystalline structure of the bulk material, and it is difficult to differentiate

304 between paracrystalline layers and crystalline cores on XRD. The presence of paracrystalline

305 cellulose, a transition phase between crystalline and amorphous region, is a recognized factor

306 interfering the accurate measurement of $\mathrm{CrI}$ and crystallite structure parameters (Park et al.,

307 2010; Nishiyama et al., 2012). A relationship between d-spacing values in (200) planes $\left(d_{200^{-}}\right.$

308 spacing) in cellulose $\mathrm{I}_{\beta}$ and the amount paracrystalline cellulose was investigated. An increased

309 amount paracrystalline cellulose layers will yield larger $d_{200}$-spacing while a perfect cellulose

310 crystallite has a minimal value perhaps between $0.384 \mathrm{~nm}-0.3866 \mathrm{~nm}$ (Ioelovich et al., 2010;

311 Nishiyama et al. 2002). The $\mathrm{d}_{200}$-spacing of $\mathrm{NCC}$ is clearly smaller than that of Avicel

312 confirming NCC is a substrate more closely representing compact cellulose crystallite. Table 2

313 also showed the crystallite size obtained from XRD analysis. NCC has a higher CrI with a smaller

314 crystallite size compared to Avicel. This is consistent with the closer $d$-spacing values and a more

315 compact crystallite structure of NCC. This result also suggests that amorphous and crystalline cellulose

316 regions are not starkly divided, further supporting the presence of paracrystalline cellulose in cellulose

317 macromolecules. A model of cellulose crystallite structure from I $\alpha$ with 36 glucan chains has been

318 previous illustrated (Lehtio et al., 2010). Based on the data obtained from this study, a similar 36

319 glucan chains arrangement for cellulose $\mathrm{I}_{\beta}$ is proposed as shown in Figure 6. Each block presents

320 one glucan chain in the crystallite with c pointing to the axis of glucan chains (French 2014). 


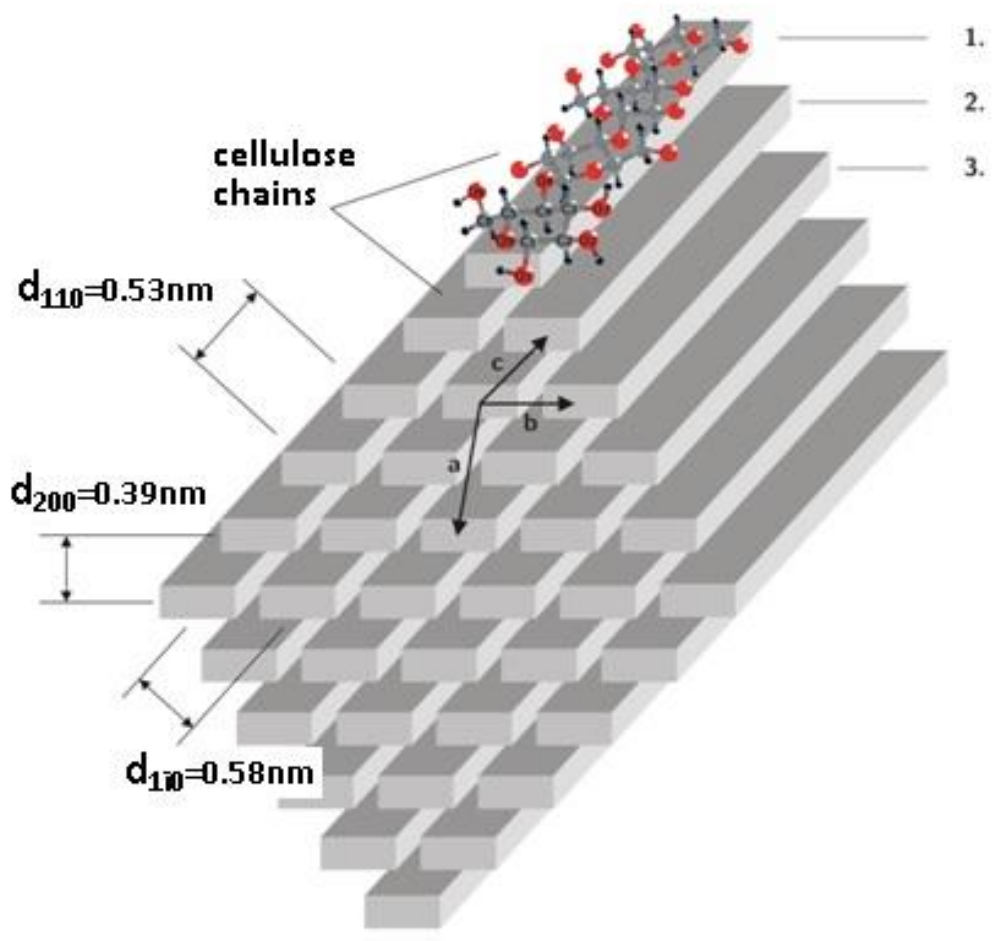

322 Figure 6. Proposed crystallite structure of nanocrystalline cellulose

\section{Conclusions}

In conclusion, we have shown an improved XRD method for better quantification of

326 cellulose CrI. The method utilizes a simple 3-parameter background model obtained from a

327 representative crystalline sample and extension of the $2 \theta$ angle to $75^{\circ}$. This new method uses

328 three distinct amorphous peaks identified from representative amorphous samples to calculate

329 CrI instead of using only one peak representative amorphous peak. This method enables a more

330 reliable measurement of CrI of cellulose $\mathrm{I}_{\beta}$ as well as d-spacing and crystallite size of cellulose

331 crystallites, offering a useful tool to investigate plant cellulose crystallite structural changes

332 during various biological and chemical treatments. The new background and amorphous peaks

333 treatment procedures developed in this study may also be applied to different cellulose

334 polymorphs to improve the quantification of $\mathrm{CrI}$. 


\section{5. Acknowledgments}

$336 \quad$ Funding for this research was provided by National Science Foundation (award number

337 1067012). The $X$-ray diffraction research was performed in EMSL, a national scientific user

338 facility sponsored by the U. S. Department of Energy's Office of Biological and Environmental

339 Research and located at Pacific Northwest National Laboratory in Richland, Washington. 
342 Agarwal, U.P., Reiner, R.S. \& Ralph, S.A. (2010). Cellulose I crystallinity determination using 343 FT-Raman spectroscopy: univariate and multivariate methods. Cellulose, 17, 721-733.

344 Ai-Zuhair, S. (2008). The effect of crystallinity of cellulose on the rate of reducing sugars production by heterogeneous enzymatic hydrolysis. Bioresource Technology, 99, 4078-4085. wood and the size of cellulose crystallites in Norway spruce (Picea abies). Journal of Wood Science, 49, 531-537.

Bansal, P., Hall, M., Realff, M.J., Lee, J.H. \& Bommarius, A.S. (2010). Multivariate statistical analysis of X-ray data from cellulose: A new method to determine degree of crystallinity and predict hydrolysis rates. Bioresource Technology, 101, 4461-4471.

Cao, Y. \& Tan, H.M. (2005). Study on crystal structures of enzyme-hydrolyzed cellulosic materials by X-ray diffraction. Enzyme and Microbial Technology, 36, 314-317.

Chanzy, H., Marechal, Y. \& Nishiyama, Y. (1999). Toward a better knowledge of the crystalline structure of cellulose. Abstracts of Papers of the American Chemical Society, 217, U264-U264.

Deraman, M., Zakaria, S. \& Murshidi, J.A. (2001). Estimation of crystallinity and crystallite size of cellulose in benzylated fibres of oil palm empty fruit bunches by X-ray diffraction. Japanese Journal of Applied Physics Part 1-Regular Papers Short Notes \& Review Papers, 40, 3311-3314.

Dollase, W. (1986). Correction of intensities for preferred orientation in powder diffractometry: application of the March model. Journal of Applied Crystallography, 19, 267-272.

Dong, X.M., Revol, J.F. \& Gray, D.G. (1998). Effect of microcrystallite preparation conditions on the formation of colloid crystals of cellulose. Cellulose, 5, 19-32.

364 Driemeier, C. \& Calligaris, G.A. (2011). Theoretical and experimental developments for accurate determination of crystallinity of cellulose I materials. Journal of Applied Crystallography, 44, 184-192.

Evans, R., Newman, R.H., Roick, U.C., Suckling, I.D. \& Wallis, A.F.A. (1995). Changes in Cellulose Crystallinity during Kraft Pulping - Comparison of Infrared, X-Ray-Diffraction and

370 French, A.D. \& Santiago Cintrón, M. (2013). Cellulose polymorphy, crystallite size, and the 371 Segal Crystallinity Index. Cellulose, 20, 583-588.

372 French AD (2013) Idealized powder diffraction patterns for cellulose polymorphs. Cellulose, 373 21:885-896. 
Fernandes A. N., Thomas L. H., Altaner C. M., Callow P., Forsyth V. T., Apperley D. C.,

Kennedy C. J. \& Jarvis M. C. (2011) Nanostructure of cellulose microfibrils in spruce wood. Proc. Natl Acad. Sci. USA 108, E1195-E1203.

Garvey, C.J., Parker, I.H. \& Simon, G.P. (2005). On the interpretation of X-ray diffraction powder patterns in terms of the nanostructure of cellulose I fibres. Macromolecular Chemistry and Physics, 206, 1568-1575.

Habibi, Y. (2014). Key advances in the chemical modification of nanocelluloses. Chemical 382 Society Reviews, 43, 1519-1542.

383 Hall, M., Bansal, P., Lee, J.H., Realff, M.J. \& Bommarius, A.S. (2010). Cellulose crystallinity 384 a key predictor of the enzymatic hydrolysis rate. Febs Journal, 277, 1571-1582. Cellulose Extraction. Canadian Journal of Chemical Engineering, 88, 392-402.

387 Ioelovich, M., Leykin, A. \& Figovsky, O. (2010) Study of cellulose paracrystallinity. 388 Bioresources 5, 1393-1407.

Ju, X., Engelhard, M. \& Zhang, X. (2013). An advanced understanding of the specific effects of xylan and surface lignin contents on enzymatic hydrolysis of lignocellulosic biomass. Bioresource Technology, 132, 137-145.

Kim, U.J., Eom, S.H. \& Wada, M. (2010). Thermal decomposition of native cellulose: Influence on crystallite size. Polymer Degradation and Stability, 95, 778-781.

394 Kljun, A., Benians, T.A.S., Goubet, F., Meulewaeter, F., Knox, J.P. \& Blackburn, R.S. (2011). 395 Comparative Analysis of Crystallinity Changes in Cellulose I Polymers Using ATR-FTIR, X-ray 396 Diffraction, and Carbohydrate-Binding Module Probes. Biomacromolecules, 12, 4121-4126.

Liitia, T., Maunu, S.L. \& Hortling, B. (2000). Solid state NMR studies on cellulose crystallinity in fines and bulk fibres separated from refined kraft pulp. Holzforschung, 54, 618-624.

Liitia, T., Maunu, S.L., Hortling, B., Tamminen, T., Pekkala, O. \& Varhimo, A. (2003). Cellulose crystallinity and ordering of hemicelluloses in pine and birch pulps as revealed by solid-state NMR spectroscopic methods. Cellulose, 10, 307-316.

Nishiyama, Y., Langan, P. \& Chanzy, H. (2002). Crystal structure and hydrogen-bonding system in cellulose I beta from synchrotron X-ray and neutron fiber diffraction. Journal of the American Chemical Society, 124, 9074-9082.

Nishiyama Y, Johnson GP, French A (2012) Diffraction from nonperiodic models of cellulose crystals. Cellulose 19(2):319-336.

Park, S., Baker, J.O., Himmel, M.E., Parilla, P.A. \& Johnson, D.K. (2010). Cellulose crystallinity index: measurement techniques and their impact on interpreting cellulase 
411 Rietveld, H. (1969). A profile refinement method for nuclear and magnetic structures. Journal of 412 Applied Crystallography, 2, 65-71.

413 Rowe, R.C., Mckillop, A.G. \& Bray, D. (1994). The Effect of Batch and Source Variation on the 414 Crystallinity of Microcrystalline Cellulose. International Journal of Pharmaceutics, 101, 169415172.

416 Ruland, W. (1961). X-ray determination of crystallinity and diffuse disorder scattering. Acta 417 Crystallographica, 14, 1180-1185.

418 Ryu, D., Lee, S.B. \& Tassinari, T. (1981). Effect of Crystallinity of Cellulose on Enzymatic419 Hydrolysis Kinetics. Abstracts of Papers of the American Chemical Society, 182, 58-Micr.

420 Segal, L., Creely, J.J., Jr, A.E.M. \& Conrad, C.M. (1959). An Empirical Method for Estimating the Degree of Crystallinity of Native Cellulose Using the X-Ray Diffractometer. Tex Res J, 29, 786-794.

Soltes, E.J. (1983). Cellulose in the Plant-Cell Wall - Crystallinity and Accessibility Issues. Abstracts of Papers of the American Chemical Society, 186, 9-Cell.

Tanahashi, M., Goto, T., Horii, F., Hirai, A. \& Higuchi, T. (1989). Characterization of SteamGakkaishi, 35, 654-662.

428 Teeaar, R., Serimaa, R. \& Paakkari, T. (1987). Crystallinity of Cellulose, as Determined by 429 Cp/Mas Nmr and Xrd Methods. Polymer Bulletin, 17, 231-237.

430 Thygesen, A., Oddershede, J., Lilholt, H., Thomsen, A.B. \& Stahl, K. (2005). On the determination of crystallinity and cellulose content in plant fibres. Cellulose, 12, 563-576.

432 Vonk, G.G. (1973). Computerization of Ruland's X-ray Method for Determination of the 433 Crystallinity in Polymers. Journal of Applied Crystallography, 6, 148-153.

434 Weimer, P.J., Hackney, J.M. \& French, A.D. (1995). Effects of Chemical Treatments and 435 Heating on the Crystallinity of Celluloses and Their Implications for Evaluating the Effect of 436 Crystallinity on Cellulose Biodegradation. Biotechnology and Bioengineering, 48, 169-178.

437 Zhang, Y.H.P., Cui, J.B., Lynd, L.R. \& Kuang, L.R. (2006). A transition from cellulose swelling 438 to cellulose dissolution by o-phosphoric acid: Evidence from enzymatic hydrolysis and 439 supramolecular structure. Biomacromolecules, 7, 644-648. 